\section{Leiomyoma of the Forehead}

Jae Hyun Kwon ${ }^{1}$, So Young $\operatorname{Lim}^{1}$, Ha Seong Lim $^{2}$

${ }^{1}$ Department of Plastic Surgery, Samsung Medical Center, Sungkyunkwan University School of Medicine, Seoul; ${ }^{2} I^{\prime} m$ Skin Clinic, Seoul, Korea

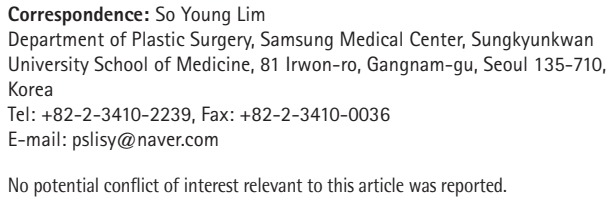

No potential conflict of interest relevant to this article was reported.

Received: 22 Nov 2012 • Revised: 24 Dec 2012 • Accepted: 9 Jan 2013 pISSN: 2234-6163 • elSSN: 2234-6171

http://dx.doi.org/10.5999/aps.2013.40.2.165 • Arch Plast Surg 2013;40:165-167 Copyright (C) 2013 The Korean Society of Plastic and Reconstructive Surgeons This is an Open Access article distributed under the terms of the Creative Commons This is an Open Access article distributed under the terms of the Creative Commons
Attribution Non-Commercial License (http://creativecommons.org/licenses/by-nc/3.0/) which permits unrestricted non-commercial use, distribution, and reproduction in any medium, provided the original work is properly cited.

Leiomyoma is a benign proliferation of smooth muscle cells that may be found in various anatomical locations. Leiomyoma of the uterus is the most common, and there have been a few case reports of extra-uterine leiomyomas including on the hand, foot, esophagus, trachea, heart, and even in vessels. However, there have been no reports of leiomyoma of the frontalis muscle. We present a case of leiomyoma of the frontalis muscle that was excised using an endoscopic method.

A 5-year-old boy was referred to our hospital with a palpable mass on his left forehead. It was first observed

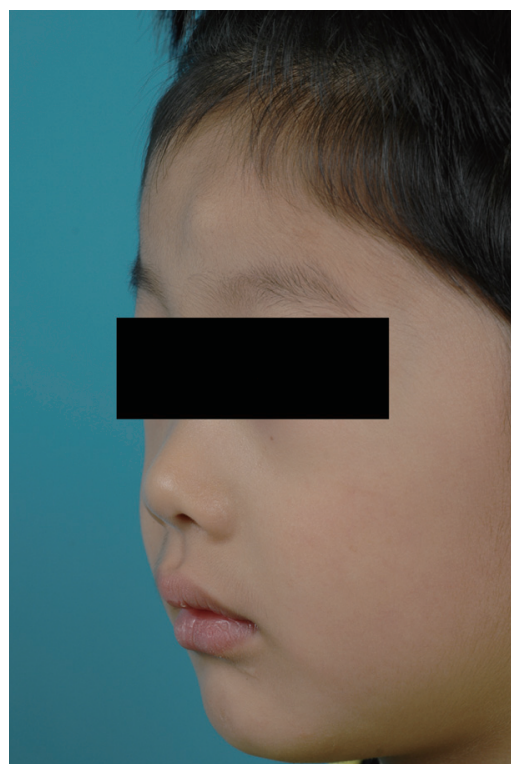

Fig. 1.

Preoperative photograph showing bulging of the left forehead due to the mass. a lipoma. when he was 4-year-old (Fig. 1). Computed tomography revealed a nonspecific soft tissue lesion on his left forehead. Except for the lesion, there were no other abnormalities on his skull, ventricle, or brain parenchyma. The patient revisited the hospital 1 year after the first visit because the size of the mass had increased.

The mass was $1 \mathrm{~cm}$ in diameter and non-tender, and the patient did not experience any discomfort. The mass was somewhat firm and movable. There were no clinical signs suggesting an inflammatory lesion such as an epidermal cyst. We initially suspected

The mass was excised using an endoscopic approach under general anesthesia. Anesthesia and hydrodissection were performed by injecting an excessive amount of lidocaine mixed with epinephrine between the galea and the periosteum, to ease the performance of the dissection. Two incisions of $1.5 \mathrm{~cm}$ in length were made $1 \mathrm{~cm}$ behind the hairline for less visible scarring and to create the shortest distance and easiest approach to the lesion. The endoscope with a retractor and other surgical instruments were inserted through these incisions. We dissected the muscle using a Freer elevator with palpation (Fig. 2). The mass was identified intramuscularly, and was completely excised without any remnant lesion. It was solid with a fibrous capsule (Fig. 3).

Pathologically, the mass showed the characteristics of a leiomyoma, and a definite diagnosis was confirmed based on the immunohistochemistry showing desmin

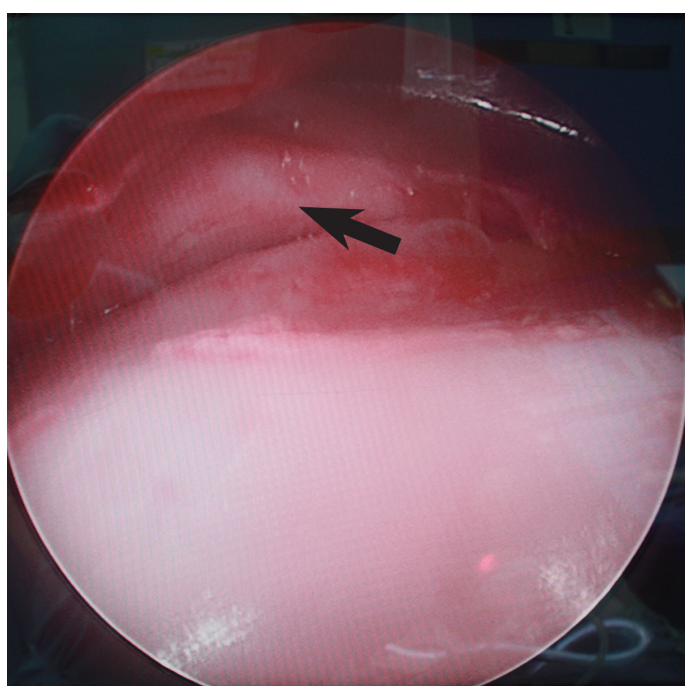

Fig. 2.

Intraoperative endoscopic image showing the mass (black arrow) in the frontalis muscle. 
positivity (Fig. 4), because desmin is a specific protein that is expressed in smooth and skeletal muscle myocytes and is also expressed in neoplasms with smooth or skeletal muscle differentiation.

Usually, leiomyomas originate from the muscle of the uterus and alimentary tract, but it is rare for a neoplasm to originate from the soft tissue of the head or neck. Benign smooth muscle tumors that arise from the forehead are rare, and this is the first case of leiomyoma in the forehead [1-3]. Among the soft tissue tumors in the head and neck area, 96\% are known to be benign [4], and leiomyomas comprise only $1.3 \%$ of them; no cases of forehead leiomyoma have been published.

A relationship between leiomyoma and trauma has been discussed in a previous study [1]. In our case, however, there was no history of trauma in the years just before the tumor was recognized.

A precise pathological examination is required before surgery in forehead leiomyoma cases, even if the tumor is believed to be benign, because the examination can precisely represent the character of the lesion. A specimen is assumed to be malignant if an average of one or more mitoses in every five highpower fields is identified. Moreover, if mitoses are found in every high-power field, the specimen is considered to be definitely malignant [3]. The pathologic study of this case demonstrated an average of less than one mitosis in every five high-power fields; therefore, the specimen had a high likelihood of being a benign leiomyoma.
The definitive treatment for subcutaneous leiomyomas of the forehead and other body parts is complete excision of the lesion. However, the choice of surgical method, approach, and extent may vary according to the site in which the tumor is involved. In our case, total excision of the mass was achieved via an endoscopic approach. Because the procedure is minimally invasive, the patient was discharged on postoperative day 1 with a mild compressive dressing to minimize the swelling, and the wound healed uneventfully; the scars were almost unidentifiable (Fig. 5).

Leiomyoma is a benign proliferation of smooth muscle cells that may be found in various anatomical locations. The disease is not rare, but most cases are focused on the uterus. An extra-uterine leiomyoma typically presents as a small, painless mass. This article is meaningful in that it shows leiomyoma can occur on the frontalis muscle. Magnetic resonance imaging may be helpful for diagnosis, but a definite diagnosis is confirmed by pathology [5]. Simple excision yields excellent results, but to minimize scarring, an endoscopic approach should be used. In addition, because cases of multiple metastasizing leiomyoma have been reported, regular follow-up should be considered.

\section{References}

1. Haedicke G, Kaban LB. Smooth-muscle tumors of the oral cavity. Plast Reconstr Surg 1988;81:264-9.

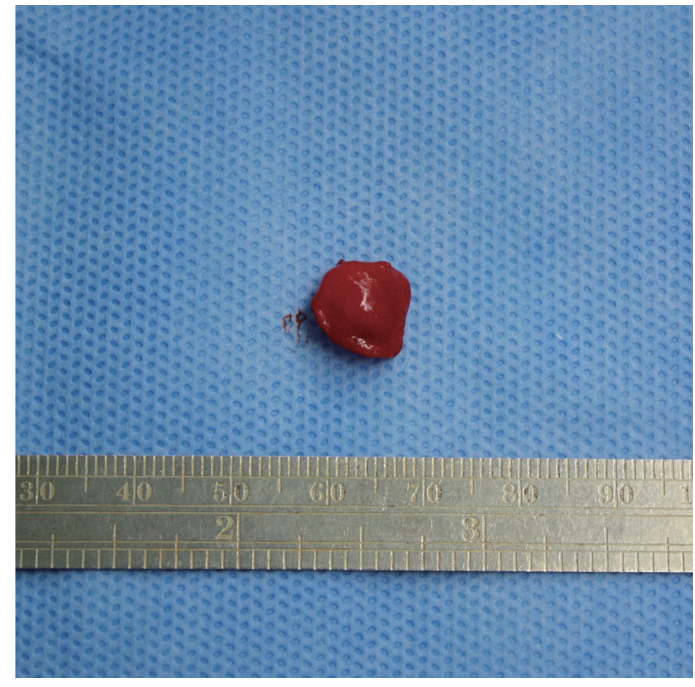

Fig. 3. Excised specimen showing a solid mass with a fibrous capsule.

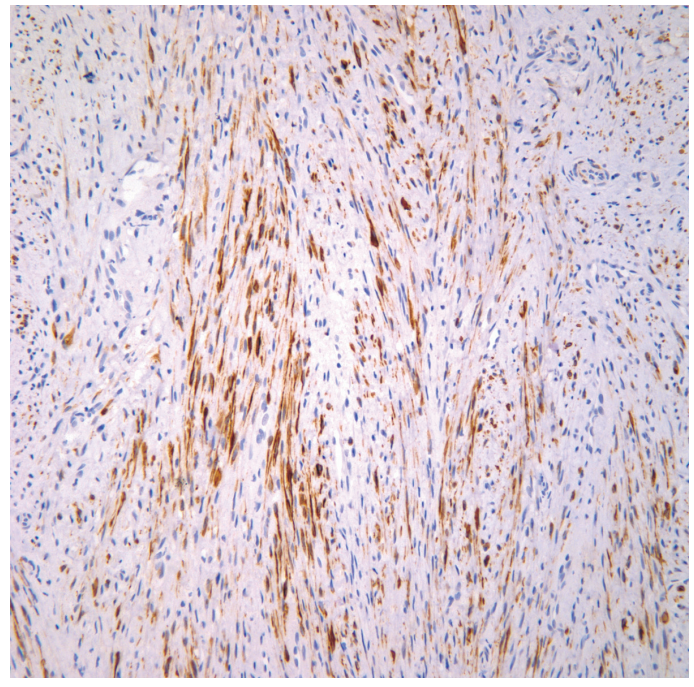

Fig. 4. Desmin stained histology of the patient, showing that the origin of this mass was the smooth muscle $(\times 100)$ 
2. Mindell RS, Calcaterra TC, Ward PH. Leiomyosarcoma of the head and neck: a review of the literature and report of two cases. Laryngoscope 1975;85:904-10.

3. Stout AP, Hill WT. Leiomyosarcoma of the superficial soft tissues. Cancer 1958;11:844-54.

4. Makino Y. A clinicopathological study on soft tissue tumors of the head and neck. Acta Pathol Jpn 1979; 29:389-408.

5. Wongsripuemtet J, Ruangchira-urai R, Stern EJ, et al. Benign metastasizing leiomyoma. J Thorac Imaging 2012;27:W41-3.

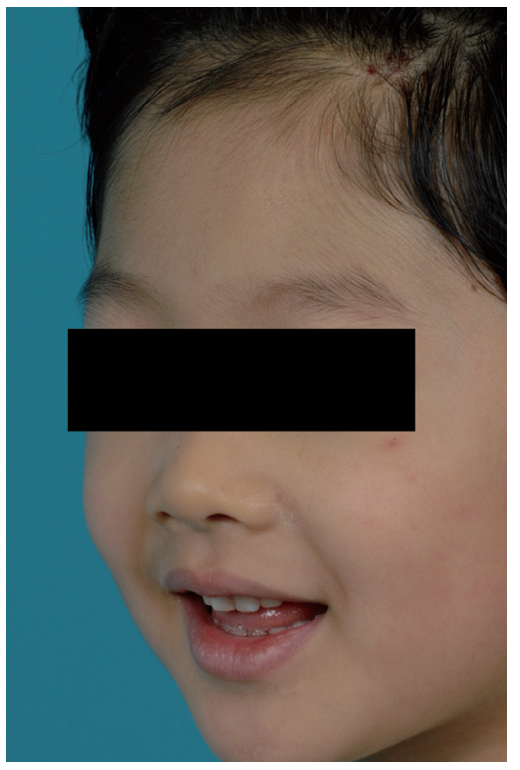

Fig. 5.

Follow-up photograph at 10 days postoperatively. 